Pesq. Vet. Bras. 35(3):209-215, março 2015 DOI: $10.1590 / \mathrm{S} 0100-736 \mathrm{X} 2015000300001$

\title{
Sinais clínicos e patologia da intoxicação crônica experimental de caprinos por Palicourea marcgravii ${ }^{1}$
}

\author{
Edson de F.G. Barbosa ${ }^{2}$, Saulo P. Cardoso ${ }^{2}$, Sérgio L. Salomon Cabral Filho ${ }^{3}$, José \\ Renato Junqueira Borges ${ }^{2}$, Eduardo M. Mendes de Lima², Franklin Riet-Correa ${ }^{4}$ \\ e Márcio B. Castro ${ }^{2 *}$
}

\begin{abstract}
Barbosa E.F.G., Cardoso S.P., Cabral Filho S.L.S., Borges J.R.J., Lima E.M.M., Riet-Correa F. \& Castro M.B. 2015. [Clinical signs and pathology of chronic experimental poisoning by Palicourea marcgravii in goats.] Sinais clínicos e patologia da intoxicação crônica experimental de caprinos por Palicourea marcgravii. Pesquisa Veterinária Brasileira 35(3):209-215. Laboratório de Patologia Veterinária, Hospital Veterinário, Universidade de Brasília, Via L4 Norte, Cx. Postal 4508, Brasília, DF 70910-970, Brazil. E-mail: mbcastro@unb.br

Palicourea marcgravii is considered the main toxic plant for livestock in Brazil, however, only the acute intoxication has been reported. This study reports the clinical and pathological changes of 10 goats chronically intoxicated by P. marcgravii. The animals received, daily, $0.2 \mathrm{~g} / \mathrm{kg}$ body weight of the fresh plant during 6 to 38 days. The main clinical signs were anorexia, lethargy, tachycardia, arrhythmia, positive jugular venous pulse and recumbency. At necropsies, the lesions were pale kidneys and myocardium, serous atrophy of cardiac fat, evident hepatic lobular pattern and pulmonary edema. The main microscopic findings were swelling and vacuolization of cardiomyocytes, cardiac necrosis and inflammatory mononuclear infiltrate of the myocardium. The kidneys showed diffuse vacuolar hydropic degeneration of the epithelium. These findings demonstrated that goats chronically intoxicated by $P$. marcgravii show distinct clinicopathologic features than those observed in the acute form of the poisoning. It is suggested that goats may be spontaneously become intoxicated by the ingestion of small repeated doses of the plant and that the poisoning can be confused with other diseases.
\end{abstract}

INDEX TERMS: Poisonous plants, Palicourea marcgravii, Rubiaceae, experimental plant poisoning, cardiotoxicity, goats.

RESUMO.- Palicourea marcgravii é considerada a principal planta tóxica de interesse pecuário no Brasil, porém, até o momento, é conhecido apenas o quadro agudo da intoxicação. Esse estudo avaliou as alterações clínico-patológicas de 10 caprinos cronicamente intoxicados por $P$. marcgravii. Os animais receberam, diariamente, $0,2 \mathrm{~g} / \mathrm{kg}$ de peso corporal da planta fresca por 6 a 38 dias. Os caprinos apresentaram como principais sinais clínicos anorexia, apatia,

\footnotetext{
${ }^{1}$ Recebido em 2 de junho de 2014.

Aceito para publicação em 9 de setembro de 2014.

${ }^{2}$ Hospital Veterinário, Universidade de Brasília (UnB), Via L4 Norte, Cx. Postal 4508, Brasília, DF 70910-970, Brasil. *Autor para correspondência: mbcastro@unb.br

${ }^{3}$ Centro de Manejo de Ovinos, Fazenda Água Limpa, UnB, Brasília, DF 71750-000.

${ }^{4}$ Hospital Veterinário, Universidade Federal de Campina Grande, Patos, PB 58700-000, Brasil.
}

taquicardia, arritmia, pulso venoso jugular positivo e decúbito. Nas necropsias, os achados macroscópicos foram hidropericárdio, palidez dos rins e do miocárdio, atrofia gelatinosa da gordura cardíaca, evidenciação do padrão lobular hepático e edema pulmonar. Os principais achados microscópicos foram tumefação e vacuolização de cardiomiócitos, necrose de fibras cardíacas e infiltrado inflamatório mononuclear no miocárdio. Nos rins foi encontrada marcante degeneração hidrópico-vacuolar difusa. Os achados demonstraram nos caprinos cronicamente intoxicados, quadro clínico-patológico com características distintas do observado na forma aguda da intoxicação por P. marcgravii. Essas observações comprovam o risco para caprinos da ingestão da planta, mesmo que em pequenas doses, resultando no surgimento de quadro clínico e graves lesões ainda pouco conhecidas, e que poderiam ser confundidas com outras enfermidades. 
TERMOS DE INDEXAÇÃO: Plantas tóxicas, Palicourea marcgravii, Rubiaceae, intoxicação experimental por planta, cardiotoxicidade, caprinos.

\section{INTRODUÇÃO}

Palicourea marcgravii é considerada a principal planta tóxica de interesse pecuário no Brasil, encontrando-se distribuída por quase todo o País, com exceção do Mato Grosso do Sul e da Região Sul. Na região Nordeste, somente foi descrita em áreas com boa pluviosidade, não ocorrendo no semiárido, nem em várzeas (Tokarnia et al. 2012). Sua importância é reforçada pela extensa distribuição, boa palatabilidade, elevada toxidez e efeito acumulativo, sendo responsável pela morte de $80 \%$ dos bovinos intoxicados por plantas na região amazônica (Tokarnia et al 1986, 1991, 2012). As folhas frescas de Palicourea marcgravii contem ácido monofluoroacético (MFA) e causam morte súbita de animais, mesmo após a ingestão de uma única dose de 0,6 e $1,0 \mathrm{~g} / \mathrm{kg}$ para bovinos e caprinos, respectivamente (Tokarnia \& Döbereiner 1986, Tokarnia et al. 1991).

No Distrito Federal (DF) foi observada a morte de caprinos com sinais neurológicos, mantidos em áreas com acesso à matas ciliares, que continham pequenas quantidades de $P$. marcgravii e com sinais de ingestão da planta pelos animais. Na época, foi feito o diagnóstico histológico de polioencefalomalácia. Essa lesão havia sido relatada anteriormente em bovinos com suspeita de intoxicação pela planta (Ecco et al. 2004). Esses achados motivaram estudo piloto prévio em caprinos com doses baixas e repetidas $(0,2 \mathrm{~g} / \mathrm{kg}$ de peso vivo de folhas frescas da planta tóxica), onde não foi detectada nenhuma alteração no SNC, no entanto, surgiram severas lesões degenerativo-necróticas no miocárdio com a morte dos animais.

Até o momento, a maior parte dos estudos com animais de produção visaram determinar o efeito e a determinação da dose tóxica de $P$. marcgravii na reprodução do quadro agudo da intoxicação, observado com frequência na maioria dos surtos (Tokarnia et al. 1986, 1990, 1991, Barbosa et al. 2003). A ingestão de pequenas doses diárias de $P$. marcgravii, por períodos prolongados, em áreas com pouca disponibilidade da planta, poderia estar envolvida na morte de animais, principalmente por lesões cardíacas severas, diferentemente da forma aguda da intoxicação. É importante também considerar, que pequenas doses de plantas que contém MFA vem sendo usadas para induzir a resistência de ruminantes, na tentativa de prevenir intoxicações (Oliveira et al. 2013, Duarte et al. 2014) mas poderiam causar de alguma forma, lesão cardíaca aos animais.

Este trabalho teve como objetivo investigar a evolução e o efeito clínico-patológico da administração crônica de folhas frescas de P. marcgravii para caprinos.

\section{MATERIAL E MÉTODOS}

O experimento foi realizado na Fazenda Água Limpa da Universidade de Brasília, no Centro de Manejo de Ovinos, no período de agosto a outubro de 2013. Foram utilizados 13 caprinos, clinicamente sadios, sete destes da raça Parda Alpina, 4 fêmeas e 3 machos. 0 restante dos animais eram todos machos da raça Saanem e idade variando entre 6 a 8 meses. Os caprinos eram mantidos em baias coletivas cobertas, com piso de cimento e estrados de madeira, alimentados com silagem de milho, concentrado comercial para caprinos, capim elefante (Pennisetum purpureum) picado fresco, sal mineral comercial para caprinos e água fresca $a d$ libitum. Previamente ao experimento, receberam doses mensais de anti-parasitário (Closantel e Albendazol) durantes o período de adaptação alimentar e ambiental.

Dez caprinos ( $\mathrm{N}^{\mathrm{os}} 1$ a 10$)$ receberam doses orais individuais diárias de 0,2 g/kg/animal de folhas frescas de Palicourea marcgravii. As plantas foram colhidas semanalmente nas matas do Distrito Federal e Entorno, em propriedades onde anteriormente ocorreram casos de intoxicação em bovinos. Posteriormente, as plantas foram armazenadas em sacos plásticos refrigeradas por $5-8^{\circ} \mathrm{C}$ até o momento da administração para os caprinos. Os animais controle ( $\mathrm{N}^{\text {os }} 11$ a 13) foram mantidos nas mesmas condições de ambiente e alimentação, porém sem a ingestão da planta tóxica. Foi realizada avaliação clínica diária matinal, determinados o início e a duração dos sinais clínicos nos animais, com ênfase na atitude (anorexia e apatia), frequência e alterações cardíacas, frequência respiratória, vasos episclerais e mucosas, estase jugular e decúbito. 0 exame clínico foi feito até o dia anterior à eutanásia ou da morte do animal.

Durante o experimento, todos os caprinos que se apresentavam na eminência de morrer, foram eutanasiados por overdose de barbitúrico (Concea 2013). Os caprinos 3 e 7, que não demostraram sinais após 38 dias de ingestão da planta, receberam na sequência, dose única de $2 \mathrm{~g} / \mathrm{kg}$ de folhas frescas da planta, com o objetivo de determinar se os mesmos haviam desenvolvido resistência ao princípio tóxico (Oliveira et al. 2013). 0 Caprino 3 foi desafiado no dia seguinte ao término do protocolo experimental inicial e o Caprino 7, após intervalo de 5 dias. Os caprinos controle foram submetidos a eutanásia após 39 dias de experimento. Todos os animais foram necropsiados e colhidos fragmentos de órgãos (coração, pulmões, fígado, rins, adrenal, baço, sistema nervoso central, intestinos, pâncreas, linfonodos e músculo estriado esquelético) e fixados em solução de formol tamponado a $10 \%$. As amostras histológicas foram processadas rotineiramente e confeccionados cortes histológicos corados pela técnica hematoxilina-eosina (HE) para avaliação em microscopia de luz.

\section{RESULTADOS}

Nos caprinos cronicamente intoxicados por Palicourea marcgravii o início dos sinais clínicos ocorreu entre 5 a 6 dias após início do experimento; entretanto, oito dos 10 caprinos apresentaram alterações entre 13 e 20 dias. Oito

Quadro 1. Intoxicação crônica experimental com doses diárias de 0,2 kg/kg de Paliourea marcgravii em caprinos

\begin{tabular}{|c|c|c|c|c|c|c|}
\hline Caprino & Sexo & $\begin{array}{c}\text { Dias de } \\
\text { ingestão }\end{array}$ & Dose total & $\begin{array}{c}\text { Início } \\
\text { dos sinais }\end{array}$ & $\begin{array}{l}\text { Curso } \\
\text { clínico }\end{array}$ & Desfecho \\
\hline 1 & $\mathrm{M}^{\mathrm{a}}$ & 23 & $4,6 \mathrm{~g} / \mathrm{kg}$ & $19^{\circ}$ dia & 5 dias & $\mathrm{E}$ \\
\hline 2 & $\mathrm{~F}$ & 23 & $4,6 \mathrm{~g} / \mathrm{kg}$ & $13^{\circ}$ dia & 11 dias & $\mathrm{E}$ \\
\hline 3 & M & 38 & $7,6 \mathrm{~g} / \mathrm{kg}$ & $19{ }^{\circ} \mathrm{dia}$ & 20 dias & Mo \\
\hline 4 & M & 38 & $7,6 \mathrm{~g} / \mathrm{kg}$ & $23^{\circ} \mathrm{dia}$ & 16 dias & $\mathrm{E}$ \\
\hline 5 & M & 38 & $7,6 \mathrm{~g} / \mathrm{kg}$ & $6^{\circ}$ dia & 33 dias & $\mathrm{E}$ \\
\hline 6 & M & 16 & $3,2 \mathrm{~g} / \mathrm{kg}$ & $16^{\circ}$ dia & 1 dia & Mo \\
\hline 7 & M & 38 & $7,6 \mathrm{~g} / \mathrm{kg}$ & $26^{\circ}$ dia & 13 dias & Mo \\
\hline 8 & M & 30 & $6,0 \mathrm{~kg} / \mathrm{kg}$ & $17^{\circ} \mathrm{dia}$ & 14 dias & $\mathrm{E}$ \\
\hline 9 & M & 6 & $1,2 \mathrm{~g} / \mathrm{kg}$ & $5^{\circ}$ dia & 2 dias & Mo \\
\hline 10 & M & 27 & $5,4 \mathrm{~g} / \mathrm{kg}$ & $20^{\circ}$ dia & 8 dias & Mo \\
\hline 11 & $\mathrm{~F}$ & \multicolumn{4}{|c|}{ Controle } & $\mathrm{E}$ \\
\hline 12 & $\mathrm{~F}$ & \multicolumn{4}{|c|}{ Controle } & $\mathrm{E}$ \\
\hline 13 & $\mathrm{~F}$ & \multicolumn{4}{|c|}{ Controle } & E \\
\hline
\end{tabular}

$\overline{\mathrm{a}} \mathrm{M}=$ macho, $\mathrm{F}$ = fêmea, $\mathrm{E}=$ eutanásia, $\mathrm{Mo}=$ morte espontânea. 
Quadro 2. Alterações histopatológicas cardíacas em caprinos cronicamente intoxicados com Palicourea marcgravii

\begin{tabular}{|c|c|c|c|c|c|c|c|c|c|c|c|c|c|c|}
\hline \multirow[t]{2}{*}{ Animal } & \multirow{2}{*}{\multicolumn{3}{|c|}{$\frac{\text { Átrio direito }}{\text { Necr }^{\mathrm{a}} \text { Deg Infl }}$}} & \multicolumn{3}{|c|}{ Átrio esquerdo } & \multicolumn{3}{|c|}{$\begin{array}{c}\text { Septo } \\
\text { interventricular }\end{array}$} & \multicolumn{3}{|c|}{$\begin{array}{c}\text { Ventrículo } \\
\text { direito }\end{array}$} & \multicolumn{2}{|c|}{$\begin{array}{l}\text { Ventrículo } \\
\text { esquerdo }\end{array}$} \\
\hline & & & & & & & $\mathrm{Necr}$ & Deg & Infl & Necr & Deg & Infl & $\mathrm{Necr}$ & Deg Infl \\
\hline 1 & + & + & - & - & ++ & + & ++ & ++ & + & + & + & + & + & $+++\quad+$ \\
\hline 2 & + & + & + & + & ++ & + & + & +++ & + & + & +++ & + & + & $+++\quad+$ \\
\hline 3 & + & + & - & - & - & - & - & + & - & + & + & + & + & + \\
\hline 4 & - & + & ++ & - & + & + & + & +++ & + & + & +++ & + & + & $+++\quad+$ \\
\hline 5 & - & + & + & + & + & + & + & ++ & + & + & +++ & + & + & ++ \\
\hline 6 & + & + & + & + & + & + & ++ & +++ & + & ++ & +++ & + & + & $+++\quad+$ \\
\hline 7 & - & - & - & - & + & - & + & + & - & + & + & - & + & + \\
\hline 8 & - & - & + & - & + & + & + & +++ & + & + & + & + & + & $+++\quad+$ \\
\hline 9 & + & - & + & + & + & + & +++ & ++ & + & ++ & + & + & ++ & $++\quad++$ \\
\hline 10 & + & + & - & + & + & - & ++ & +++ & + & + & + & + & + & $+++\quad+$ \\
\hline 11 & - & - & - & - & - & - & - & - & - & - & - & - & - & - \\
\hline
\end{tabular}

${ }^{a}$ Necr = necrose individual de cardiomiócitos, Deg = tumefação e vacuolização de cardiomiócitos, Infl = Infiltrado inflamatório mononuclear; - sem alterações, + discreta,++ moderada,+++ acentuada.

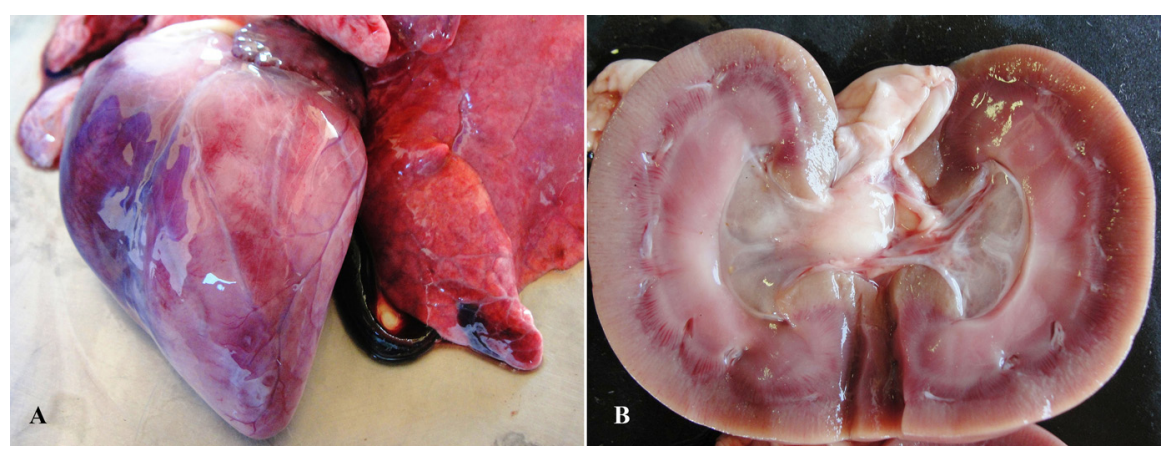

Fig.1. Lesões macroscópicas em caprinos cronicamente intoxicados por Palicourea marcgravii. (A) Palidez acentuada, multifocal a coalescente no miocárdio. (B) Intensa palidez difusa renal.

caprinos receberam a planta por 23 a 38 dias, um ingeriu a mesma por somente seis dias e outro por 16 dias (Quadro 1). A dose mínima total de planta ingerida que causou a morte de um caprino $\left(\mathrm{N}^{\circ}\right.$ 9) em apenas 6 dias foi de $1,2 \mathrm{~g} /$ $\mathrm{kg}$. Porém, a maioria dos animais recebeu doses totais de 4,6-7,6g/kg até o desfecho do experimento (Quadro 1). Os sinais clínicos surgiram de forma gradativa e progressivamente se agravavam até a morte ou eutanásia do animal. Os Caprinos $\mathrm{N}^{\text {os }} 3,4,5$ e 7, que receberam a planta por mais tempo (38 dias), apresentaram manifestações clínicas semelhantes às dos outros caprinos, porém estabilizaram até o final do protocolo experimental. Todos os animais cronicamente intoxicados apresentaram taquicardia, arritimia, taquipnéia, vasos episclerais ingurgitados e decúbito esternal. Nove caprinos apresentaram apatia $\left(\mathrm{N}^{\text {os }} 1\right.$, 2, e 3-10) e pulso jugular positivo ( ${ }^{\text {os }} 1-8$ e 10$)$ e oito apresentaram anorexia $\left(\mathrm{N}^{\circ \mathrm{s}} 1,2,4,6-8\right.$ e 10). 0 Caprino 3 que recebeu desafio de $2 \mathrm{~g} / \mathrm{kg}$ de folhas frescas da planta após 24 horas do término do experimento, apresentou sinais clínicos semelhantes aos outros animais, porém, três horas após a dose, entrou em decúbito e permaneceu apático até a morte. 0 Caprino 7, ao ser desafiado com $2 \mathrm{~g} / \mathrm{kg}$ de folhas frescas da planta, 5 dias após a última administração, não apresentou sinais clínicos durante 12 horas de observação; entretanto, foi encontrado morto 12 horas após.

As principais alterações macroscópicas nos animais do experimento foram observadas no coração, rim, fígado e pulmões. As lesões cardíacas mais significativas foram palidez do miocárdio nos 10 caprinos (Fig.1A), hidropericárdio em nove ( $\mathrm{N}^{\text {os }} 1-8$ e 10) e atrofia gelatinosa da gordura em três ( $\mathrm{N}^{\circ \mathrm{s}} 1,6$ e 10). Os rins de todos os caprinos apresentavam-se pálidos (Fig.1B) e no fígado havia padrão lobular evidenciado e congestão difusa em todos os animais, exceto no caprino 11 . Os pulmões de 9 ( $\left.\mathrm{N}^{\text {os }} 1-9\right)$ apresentavam edema leve a moderado e em cinco ( $\mathrm{N}^{\text {os }} 1-3,6$ e 7 ) havia congestão. Os animais controle não apresentaram alterações macroscópicas dignas de nota.

0 exame histopatológico de todos os caprinos intoxicados demonstrou alterações principalmente no coração (Quadro 2). Observou-se severa tumefação e vacuolização de cardiomiócitos (Fig.2A) com distribuição multifocal à difusa. As fibras cardíacas apresentavam expansão e vacuolização do citoplasma, caracterizada por marcada área perinuclear não corada. Os caprinos 3 e 7 apresentaram apenas tumefação e vacuolização discreta e focal de cardiomiócitos. Outro achado foi a necrose individual de cardiomiócitos, com distribuição esparsa e geralmente de intensidade discreta, caracterizada por hipereosinofilia citoplasmática, perda das estriações e, por vezes, cariopicnose. Ao redor dos focos de necrose do miocárdio, observou-se infiltrado inflamatório intersticial ou perivascular mononuclear, geralmente discreto (Fig.2B). No ventrículo esquerdo do Ca- 

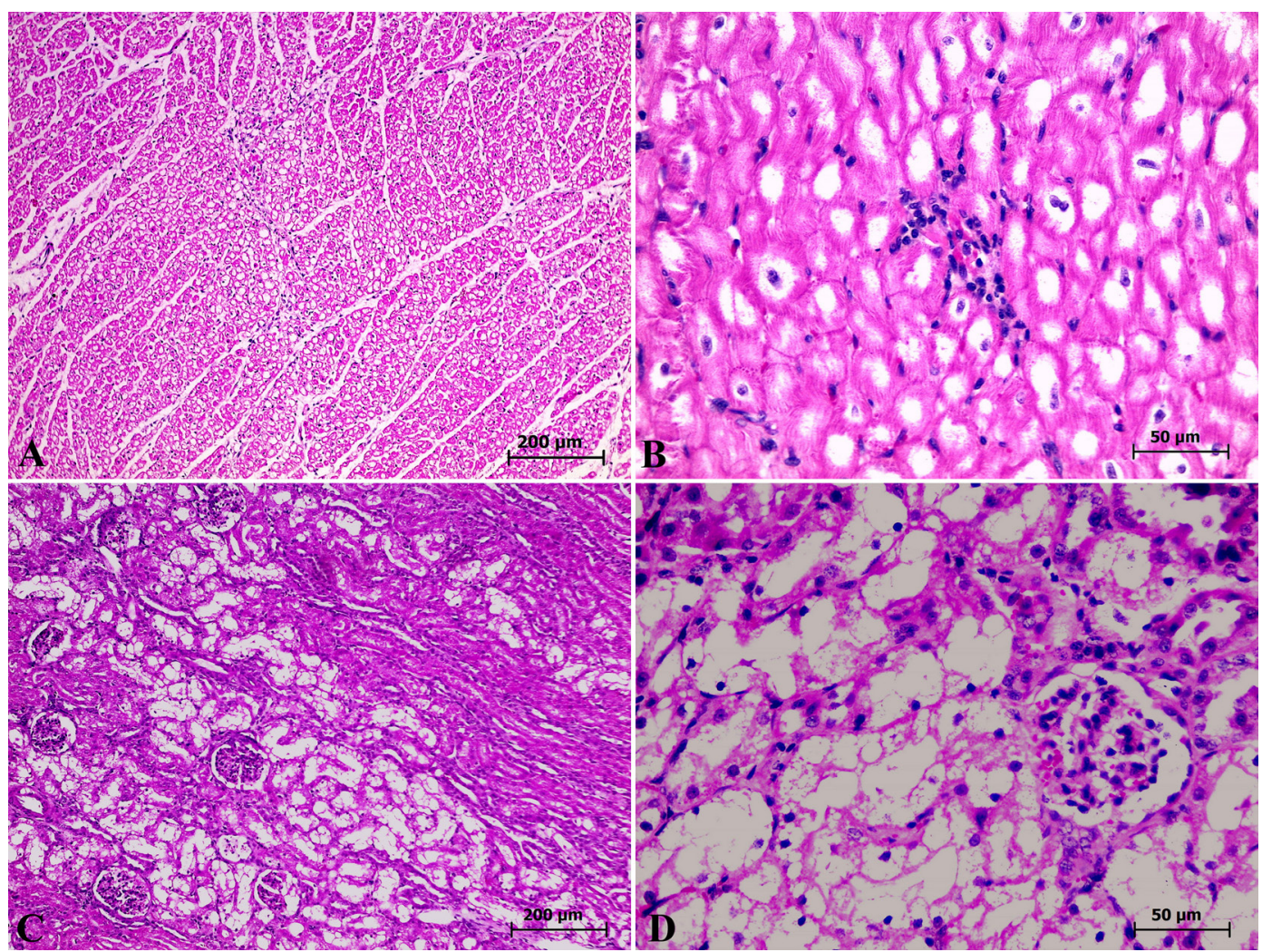

Fig.2. Alterações histopatológicas em caprinos cronicamente intoxicados por Palicourea marcgravii. (A) Coração: vacuolização multifocal a coalescente de cardiomiócitos. (B) Coração: tumefação, vacuolização de cardiomiócitos e infiltrado mononuclear discreto intersticial. (C) Rim: degeneração hidrópico-vacuolar tubular multifocal acentuada. (D) Rim: cariopicnose do epitélio renal e intensa vacuolização tubular. HE.

prino 5 foi observada fibrose discreta multifocal por entre os cardiomiócitos. 0 septo interventricular e os ventrículos direito e esquerdo foram as regiões mais frequentemente acometidas e com as principais lesões apresentando maior intensidade. Os átrios direito e esquerdo também apresentaram as mesmas alterações, porém com menor frequência e intensidade (Quadro 2).

Em todos os animais intoxicados observou-se degeneração hidrópico vacuolar do epitélio tubular renal (Fig.2C,D), variando de discreta à acentuada, com distribuição multifocal à difusa. As células epiteliais tubulares apresentavam vacúolos citoplasmáticos translúcidos e núcleos em cariopicnose, cariorexia ou ausentes.

No fígado do Caprino 9 foi constatada moderada vacuolização de hepatócitos na região centrolobular. Os hepatócitos evidenciavam citoplasma levemente eosinofílico, de aspecto granular, contendo vacúolos bem delimitados, não corados. Congestão e edema pulmonar discreto foram encontrados no pulmão de alguns caprinos do experimento.

Os animais controle que foram eutanasiados não apresentaram alterações microscópicas dignas de nota em todos os tecidos analisados.

\section{DISCUSSÃO}

A ingestão de doses baixas e repetidas de Palicourea marcgravii em caprinos causou a morte, comprovando que esta espécie é sensível à intoxicação crônica, assim como ao efeito cumulativo da planta, com sinais de insuficiên- cia cardíaca semelhantes aos observados na intoxicação aguda em bovinos (Tokarnia \& Döbereiner 1986) e ovinos (Tokarnia et al. 1986). Em experimento realizado com ovinos intoxicados com MFA, o mesmo não apresentou ação cumulativa, porem os sinais clínicos e os achados histopatológicos foram semelhantes aos da intoxicação por plantas que contêm essa substância (Schultz et al. 1982). A dose diária de $0,2 \mathrm{~g} / \mathrm{kg}$ de folhas frescas de $P$. marcgravii provocou intoxicação crônica em todos os animais, causando a morte após cinco a 38 dias de ingestão. A forma aguda da intoxicação ocorre em minutos até algumas horas após a administração da planta, nos bovinos na dose a partir de $0,6 \mathrm{~g} /$ kg (Tokarnia \& Döbereiner, 1986), nos ovinos de 0,5 a 1g/ $\mathrm{kg}$ (Tokarnia et a. 1986), nos bubalinos de $3 \mathrm{~g} / \mathrm{kg}$ (Barbosa et al. 2003) e nos equinos de $0,6 \mathrm{~g} / \mathrm{kg}$ (Tokarnia et al. 1993).

Dentre os sinais clínicos observados nos caprinos cronicamente intoxicados, anorexia, apatia, taquicardia, arritmia, taquipnéia, estase venosa jugular positiva e decúbito são alterações frequentemente descritas em ruminantes intoxicados natural e experimentalmente pela planta (Pacheco \& Carneiro 1932, Tokarnia \& Döbereiner 1986, Tokarnia et al. 1991, Barbosa et al. 2003). No entanto, os caprinos apresentaram os sinais clínicos de forma gradativa e diária durante o experimento, e não sinais súbitos com alterações nervosas, como na forma aguda (Tokarnia et al. 1991). Esses resultados sugerem que a intoxicação crônica caracteriza-se por sinais cardiovasculares severos, mas sem as alterações neuromusculares como movimentos de 
pedalagem, opistótono e tremores musculares observadas na intoxicação aguda (Tokarnia et al. 1991, Soto-Blanco et al. 2004, Vasconcelos et al. 2008, Peixoto et al. 2012, Becker et al. 2013). A presença de vasos episclerais ingurgitados não é um achado relatado na intoxicação aguda de caprinos pela planta (Tokarnia et al. 1991, Oliveira et al. 2013).

Nos animais cronicamente intoxicados por P. marcgravii a principal lesão macroscópica foi a palidez cardíaca moderada a acentuada, sob a forma de estriações ou focos extensos. Lesões semelhantes foram observadas anteriormente na intoxicação aguda de caprinos por P. marcgravii (Pacheco \& Carneiro 1932, Tokarnia et al. 1991) e na toxicose crônica experimental por MFA (Schultz et al. 1982). As alterações observadas no fígado e pulmões dos caprinos cronicamente intoxicados, possivelmente são secundárias às lesões em diferentes graus encontradas no miocárdio. Isso pôde ser demonstrado pela congestão venosa (clinicamente evidenciada como estase e pulso jugular), edema e degeneração na região centro-lobular hepática (com evidenciação no padrão lobular), essa última, possivelmente em razão da hipóxia. Essas alterações podem ser observadas eventualmente em caprinos e ovinos intoxicados por plantas que contêm MFA (Tokarnia et al. 1991, Becker et al. 2013, Oliveira et al. 2013). Entretanto, não são relatadas na maioria dos casos descritos de toxicose por essas plantas em ruminantes no Brasil, possivelmente por predominar a forma aguda ou hiperaguda da intoxicação (Soto-Blanco et al. 2004, Peixoto et al. 2012, Becker et al. 2013, Oliveira et al. 2013). A palidez renal acentuada encontrada em todos os caprinos do experimento é uma alteração não descrita em ruminantes intoxicados por plantas que contêm MFA (Schultz et al. 1982, Vasconcelos et al. 2008, Peixoto et al. 2012, Oliveira et al, 2013, Duarte et al. 2014).

Histologicamente, na intoxicação experimental crônica dos caprinos por P. marcgravii, verificou-se que a região cardíaca mais comumente afetada e com lesões de maior intensidade foi o septo interventricular, seguida pelos ventrículos esquerdo e direito. Os átrios direito e esquerdo também apresentaram alterações semelhantes, porém com menor frequência e intensidade. Até o presente momento, não foram descritas lesões tão severas e com essa distribuição no coração de ruminantes intoxicados por plantas que contém MFA no Brasil, apesar da maioria dos estudos terem sido com a forma aguda da intoxicação (Vasconcelos et al. 2008, Becker et al. 2013, Peixoto et al. 2012, Oliveira et al. 2013). Não se conhece a razão de haver diferenças na distribuição e intensidade das lesões nas áreas cardíacas avaliadas dos caprinos cronicamente intoxicados. Uma possível explicação seria a existência de diferenças na taxa metabólica dos cardiomiócitos ou na permeabilidade das membranas à toxina nas regiões avaliadas, o que poderia interferir na velocidade de ação e distribuição do MFA (Buffa \& Pasquali-Ronchetti 1977).

A tumefação e vacuolização dos cardiomiócitos, com distribuição multifocal à difusa e em graus variáveis, foram as lesões preponderante em todos os caprinos intoxicados. Essas alterações foram descritas anteriormente em estudo similar com caprinos (Reis Jr et al. 2009). Na intoxicação aguda experimental de caprinos pela planta, a vacuoliza- ção dos cardiomiócitos, em grau discreto, foi relatada em menos de 30\% dos animais intoxicados (Tokarnia et al. 1991). Na intoxicação experimental de ovinos com fluoracetato de sódio foram encontradas lesões histopatológicas proeminentes caracterizadas pelo inchaço de fibras cardíacas, particularmente na região perinuclear, e perda das estriações transversais (Schultz et al. 1982). A intoxicação por MFA provoca marcado acúmulo de citrato nos tecidos, causado pelo bloqueio do ciclo de Krebs, ligado a atividade respiratória e metabólica do órgão, principalmente no miocárdio, no sistema nervoso, e em menor quantidade no fígado (Goncharov et al. 2005). Possivelmente a vacuolização dos cardiomiócitos possa ter ocorrido pela elevação do citrato tecidual, bem como pela interrupção do ciclo de Krebs, que causa uma depleção nos níveis de ATP teciduais e acúmulo de líquido intracelular. Aparentemente, ao exame na microscopia de luz, a vacuolização evidenciada nos cardiomiócitos dos caprinos sugere edema celular; entretanto, em estudo ultraestrutural do miocárdio de caprinos cronicamente intoxicados por P. marcgravii foi demonstrada a dilatação do retículo endoplasmático liso, diferentemente da lesão mitocondrial geralmente esperada (Reis Jr et al. 2009).

A necrose individual de cardiomiócitos nos caprinos cronicamente intoxicados por P. marcgravii foi outra lesão importante e com distribuição semelhante à tumefação e vacuolização dessas células. A necrose de fibras cardíacas havia sido descrita anteriormente em 50\% dos caprinos intoxicados de forma aguda pela planta (Tokarnia et al.1991). Essa lesão é observada em outros ruminantes intoxicados por plantas que contém MFA (Tokarnia et al. 1986, Oliveira et al. 2013); entretanto, não é sempre encontrada (Barbosa et al. 2003, Ecco et al. 2004, Vasconcelos et al. 2008, Becker et al. 2013). A intoxicação experimental de ovinos com MFA também provocou necrose de fibras cardíacas ao longo das paredes atriais e ventriculares. Os mecanismos responsáveis pela morte celular em animais intoxicados por MFA e plantas que o contém, ocorre devido ao comprometimento de etapas da respiração celular (Collichio-Zuanaze \& Satake 2005). Nessa situação, a morte de cardiomiócitos é um fenômeno esperado, devido à intensa resposta e susceptibilidade do coração frente à ação do MFA (Schultz et al. 1982, Goncharov et al. 2005).

0 processo inflamatório encontrado no coração dos caprinos cronicamente intoxicados pela planta também é relatado na intoxicação aguda desses animais por P. marcgravii (Tokarnia et al. 1991), em ovinos (Tokarnia et al. 1986) e em bovinos (Tokarnia \& Dobereiner 1986). Ovinos experimentalmente intoxicados por MFA também apresentaram infiltrado inflamatório mononuclear no miocárdio (Schultz et al. 1982). A migração de células inflamatórias possivelmente esteja relacionada com a necrose de fibras cardíacas por ação do MFA.

A fibrose intersticial no miocárdio, observada em um caprino, pode ser esporadicamente encontrada em ovinos e caprinos intoxicados de forma aguda pela planta (Tokarnia et al. 1986, 1991) e ocorre, provavelmente, em virtude do desaparecimento de fibras cardíacas devido à necrose e substituição por tecido fibroso (Tokarnia et al. 1991). 
Bovinos e ovinos intoxicados naturalmente por Amorimia spp., planta que também contêm MFA, apresentam necrose de cardiomiócitos, inflamação mononuclear intersticial e severa fibrose cardíaca (Soares et al. 2011, Schons et al. 2012). Essa última lesão, pode ser reproduzida experimentalmente em coelhos pela administração de $A$. exotropica por 40 dias (Soares et al. 2011). A fibrose surge nas áreas de necrose e inflamação do miocárdio, em ovinos intoxicados de forma crônica ou subaguda pelo MFA, e aparece esporadicamente na intoxicação aguda (Schultz et al. 1982). As diferenças nas lesões histológicas cardíacas nos caprinos intoxicados cronicamente por P. marcgravii e ruminantes intoxicados por Amorimia spp. poderiam ser devido à diferentes concentrações de MFA presente nos dois gêneros. P. marcgravii apresenta de $0,21 \%$ a $0,24 \%$ de MFA em sua composição, níveis muito maiores que os observados em várias espécies de Amorimia spp. que variam de 0,0007\% a 0,02\% (Lee et al. 2012). Mesmo tendo causando intoxicação crônica, as altas concentrações de MFA em P. marcgravii causaram tumefação e vacuolização de cardiomiócitos sem fibrose significante. É provável que doses menores de MFA induzam fibrose cardíaca em graus acentuados. Se considerados os níveis médios de MFA em $P$. marcgravii (Lee et al. 2012) a dose de MFA ingerida pelos caprinos cronicamente intoxicados ainda foi bem maior que aquela que causou fibrose cardíaca em ovinos experimentalmente intoxicados por MFA (Schultz et al. 1982).

A degeneração hidrópico-vacuolar difusa das células epiteliais dos túbulos renais foi observada em todos os caprinos cronicamente intoxicados. Essa alteração é frequentemente descrita em ruminantes intoxicados por $P$. marcgravii (Tokarnia et al. 1983, 1986, 1991, Tokarnia \& Döbereiner 1986, Barbosa et al. 2003, Ecco et al. 2004, Oliveira et al. 2013), por outras plantas que contém MFA (Vasconcelos et al. 2008, Becker et al. 2013) ou por MFA (Nogueira et al. 2010, Peixoto et al.2012). No entanto, essa lesão é descrita de forma focal ou multifocal, acometendo exclusivamente túbulos contorcidos distais (Tokarnia \& Döbereiner 1986, Tokarnia et al. 1986, 1991), diferentemente do observado nos caprinos deste experimento, que envolveu difusamente os túbulos do córtex renal, até a transição cortico-medular. 0 padrão encontrado de distribuição da degeneração hidrópico-vacuolar, possivelmente ocorreu devido a administração prolongada de $P$. marcgravii aos caprinos. É provável que a intoxicação crônica dos animais leve ao desenvolvimento de lesão renal mais ampla e menos seletiva que aquela encontrada na forma aguda geralmente descrita nos ruminantes. A degeneração hidrópico-vacuolar foi observada em todos os caprinos cronicamente intoxicados; entretanto na forma aguda é encontrada em apenas 36\% dos animais (Tokarnia et al. 1986). Ruminantes intoxicados por outras plantas tóxicas que possuem MFA no Brasil apresentaram degeneração hidrópico-vacuolar em 25 a 100\% dos casos (Tokarnia et al. 1983, 1986, 1991, Tokarnia \& Döbereiner, 1986, Barbosa et al. 2003, Ecco et al. 2004, , Oliveira et al. 2013). Todos os bovinos intoxicados experimentalmente por MFA demonstraram degeneração hidrópico-vacuolar nos túbulos renais contorcidos distais (Nogueira et al. 2010, Peixoto et al. 2012). As diferenças na susceptibilidade, na dose tóxica, na forma da intoxicação (aguda ou crônica) e até mesmo na planta utilizada, poderiam explicar as grandes variações encontradas na frequência e distribuição desta lesão nos animais intoxicados por plantas que contêm MFA.

A vacuolização centrolobular de hepatócitos foi um achado raro nos caprinos cronicamente intoxicados e pode ser encontrada com maior frequência na intoxicação aguda por P. marcgravii nessa espécie (Tokarnia et al. 1991). No entanto, essa lesão não foi observada em ovinos intoxicados experimentalmente por MFA (Schultz et al. 1982). A vacuolização de hepatócitos em animais intoxicados por MFA pode ou não ser relacionada ao efeito da toxina, uma vez que não se pode descartar como causa, a hipóxia associada à insuficiência cardíaca congestiva (Nogueira et al. 2010). 0 edema pulmonar foi um achado frequente nos caprinos cronicamente intoxicados pela $P$. marcgravii, e possivelmente, seja consequência de lesão e alteração funcional cardíaca produzida pela intoxicação crônica da planta.

A administração repetida de baixas doses de plantas que contêm MFA, no intuito de aumentar a concentração de bactéria rumenais produtoras de dehalogenase, vem sendo usada com sucesso na prevenção da intoxicação em ruminantes (Oliveira et al. 2013, Duarte et al. 2014). No entanto, os caprinos 3 e 7, que tinham ingerido a planta por um longo período, não resistiram ao desafio com uma dose alta da planta, independente ou não de terem passado por período de detoxificação. Uma justificativa para isso seria o fato dos animais desafiados receberem dose única elevada, diferentemente dos protocolos de indução de resistência, onde a administração era realizada por períodos alternados com 15 dias sem administração da planta (Oliveira et al. 2013, Duarte et al. 2014). De qualquer forma, é importante que seja feita a administração criteriosa de plantas que contêm MFA, mesmo quando usadas de forma preventiva, para que não ocorra a intoxicação crônica dos animais.

\section{CONCLUSÕES}

Conclui-se que baixas doses de Palicourea marcgravii, administradas por um período prolongado, são tóxicas e causam lesões cardíacas severas e morte dos caprinos.

É possível que a forma crônica da intoxicação possa ocorrer em condições de campo, especialmente quando há baixa disponibilidade da planta ou tenha sua oferta reduzida.

Mesmo a planta apresentando alta palatabilidade para ruminantes, a ingestão repetida de baixas doses, com manifestações clínicas mais discretas que na forma aguda da intoxicação, deve ser considerada no diagnóstico diferencial na morte de animais com acesso à matas com P. marcgravii.

Agradecimentos.- Ao Conselho Nacional de Pesquisa (CNPq) pelo suporte financeiro. Projeto financiado pelo Instituto de Ciência e Tecnologia para o Controle das Intoxicações por Plantas (Proc. CNPq 573534/2008-0).

\section{REFERÊNCIAS}

Barbosa J.D., Oliveira C.M.C. \& Tokarnia C.H. 2003. Comparação da sensibilidade de bovinos e búfalos à intoxicação por Palicourea marcgravii (Rubiaceae). Pesq. Vet. Bras. 23:(4):167-172. 
Becker M., Caldeira F.H.B., Carneiro F.M., Oliveira L.P., Tokarnia C.H., Riet-Correa F., Lee S.T. \& Colodel E.M. 2013. Importância da intoxicação por Amorimia pubiflora (Malpighiaceae) em bovinos no Mato Grosso do Sul: reprodução experimental da intoxicação em ovinos e bovinos. Pesq. Vet. Bras. 33:(9):1049-1056.

Buffa P. \& Pasquali-Ronchetti I. 1977. Biochemical lesions of respiratory enzymes and configurational changes of mitochondrial in vivo. Cell Tissue Res. 183:1-23.

CONCEA (Conselho Nacional de Controle de Experimentação Animal). 2013. Resolução normativa no 13, de 20 de setembro de 2013. [Ficam baixadas as Diretrizes da Prática de Eutanásia do Conselho Nacional de Controle de Experimentação Animal]. D.O.U 187:5.

Collichio-Zuanaze R.C. \& Satake M. 2005. Aspectos clínicos e terapêuticos da intoxicação por fluoroacetato de sódio em animais domésticos: revisão. Vet. Notícias 11(2):81-89.

Duarte A.L.L., Medeiros R.M.T., Carvalho F.K.L., Lee S.T., Cook D., Pfister J.A., Costa V.M.M. \& Riet-Correa F. 2014. Induction and transfer of resistance to poisoning by Amorimia (Mascagnia) septentrionalis in goats. J. Appl. Toxicol. 34(2):220-223.

Ecco R., Túry E., Santos M.M. \& Barros R.M. 2004. Intoxicação por Palicourea marcagravii em bovinos associados a malácia cerebral. Pesq. Vet. Bras. 24(Supl):20-21.

Goncharov N.V., Jenkins R.O. \& Radilov A.S. 2005. Toxicology of fluoracetate: review, with possible directions for terapy research. J. Appl. Toxicol. 26:148-161.

Lee S.T., Cook D., Riet-Correa F., Pfister J.A., Anderson W.R., Lima F.G. \& Gardner D. 2012. Detection of monofluoracetate in Palicourea and Amorimia species. Toxicon 60:791-796.

Nogueira V.A., França T.N., Peixoto T.C., Caldas S.A., Armién A.G. \& Peixoto P.V. 2010. Intoxicação experimental por monofluoroacetato de sódio em bovinos: aspectos clínicos e patológicos. Pesq. Vet. Bras. 30(7):533-540.

Oliveira M.D., Riet-Correa F., Carvalho F.K., Silva G.B., Pereira W.S. \& Medeiros R.M.T. 2013. Indução de resistência a intoxicação por Palicourea aeneosfusca (Rubiaceae) mediante administração de doses sucessivas não tóxicas. Pesq. Vet. Bras. 33(6):731-734.

Pacheco G. \& Carneiro V. 1932. Estudos experimentais sobre plantas tóxicas. I Intoxicação dos animais pela "erva de rato da mata". Revta Soc. Paulista Med. Vet. 2(2/3):23-46.

Peixoto T.C., Nogueira V.A., Caldas S.A., França T.N., Anjos B.L., Aragão A.P. \& Peixoto P.V. 2012. Efeito protetor da acetamida em bovinos indica monofluoracetato como princípio tóxico de Palicourea marcgravii (Rubiaceae). Pesq. Vet. Bras. 32(4):319-328.
Reis Jr J.L., Castro M.B., Moscardini A.C., Novais E.P.F., Borges J.R.J., Riet-Correa F. \& Howerth E.W. 2009. Histopatologic and ultrastructural findings of chronic experimental cardiotoxicity induced by monofluoracetate producing-plant Palicourea marcgravii. Proceedings of the $52^{\text {nd }}$ AAVLD Annual Conference, San Diego, CA, p.169.

Soares M.P., Pavarini S.P., Adrien M.L., Quevedo P.S., Schild A.L., Peixoto P.V., Cruz C.E.F. \& Driemeier D. 2011. Amorimia exotropica poisoning as a presumptive cause of myocardial fibrosis in cattle. J. Vet. Diagn. Invest. 23(6):1226-1229. Doi:10.1177/1040638711425586

Soto-Blanco B., Haraguchi M., Silva J.A. \& Gorniak L.S. 2004. Intoxicação natural de caprinos e ovinos por Palicourea marcgravii (Rubiaceae). Revta Caatinga 17(1):52-56.

Schons S.V., Lopes T.V., Melo T.L., Lima J.P., Riet-Correa F., Barros M.A.B. \& Schild A.L.P. 2012. Intoxicações por plantas em ruminantes e equídeos na região central de Rondônia. Ciência Rural 42(7):1257-1263.

Schultz R.A., Coetzer J.A.W., Kellerman T.S. \& Naudé T.W. 1982. Observations on the clinical, cardiac and histopatological effects in sheep. Onderstepoort J. Vet. Res. 49:237-245.

Tokarnia C.H., Döbereiner J., Couceiro J.E.M. \& Silva A.C.C. 1983. Intoxicação por Palicourea aeneofusca (Rubiaceae), a causa de "mortes súbitas" em bovinos na Zona da Mata de Pernambuco. Pesq. Vet. Bras. 3(3):7579 .

Tokarnia C.H. \& Döbereiner J. 1986. Intoxicação por Palicourea marcgravii (Rubiaceae) em bovinos no Brasil. Pesq. Vet. Bras. 6:73-92.

Tokarnia C.H., Peixoto P.V. \& Döbereiner J. 1986. Intoxicação experimental por Palicourea marcgravii (Rubiaceae) em ovinos. Pesq. Vet. Bras. 6:121-131.

Tokarnia C.H., Peixoto P.V. \& Döbereiner J. 1990. Plantas que afetam o funcionamento do coração de bovinos no Brasil. Pesq. Vet. Bras. 10:1-10.

Tokarnia C.H., Peixoto P.V. \& Döbereiner J. 1991. Intoxicação experimental por Palicourea marcgravii (Rubiaceae) em caprinos. Pesq. Vet. Bras. 11:65-70.

Tokarnia C.H., Costa E.R., Barbosa J.D., Armien A.G. \& Peixoto P.V. 1993. Intoxicação experimental por Palicourea marcgravii (Rubiaceae) em equinos. Pesq. Vet. Bras. 13:67-72.

Tokarnia C.H., Brito M.F., Barbosa J.D., Peixoto P.V. \& Döbereiner J. 2012. Plantas que afetam o funcionamento do coração, p.27-37. In: Ibid. (Eds), Plantas Tóxicas do Brasil. 2 $2^{\underline{a}}$ ed. Helianthus, Rio de Janeiro.

Vasconcelos J.S., Riet-Correa F., Dantas A.F., Medeiros R.M.T. \& Dantas A.J.A. 2008. Mortes súbitas em bovinos causadas por Palicourea aeneofusca (Rubiaceae) e Mascagnia rigida (Malpighiaceae) na Zona da Mata Paraibana. Pesq. Vet. Bras. 28:457-460. 